\title{
Understanding the Self-Assembly of a Janus-type POM-POSS Co-Cluster from Low-dose Cryo STEM
}

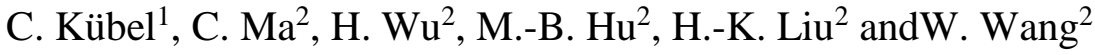 \\ 1. Karlsruhe Institute of Technology (KIT), Karlsruhe, Germany \\ 2. Nankai University, Tianjin, P. R. China
}

Understanding the self-assembly process of novel cluster-assembled materials (CAMs) is a prerequisite to optimize their structure and function. Janus-type molecules are of particular interest as they provide the possibility to combine dissimilar properties. In this study, two kinds of unlike organo-functionalized inorganic clusters (polyoxometalate: POM, polyhedral oligomeric silsesquioxane: POSS) have been covalently linked by a short organic tether to form a dumbbellshaped Janus co-cluster. Their supramolecular assembly in nanocrystals has been analyzed in 2D and 3D using low-dose (cryo) HAADF-STEM techniques to understand the assembly process based on the structure and the structural variations for various linkers.

With a short flexible $\mathrm{C}_{4}$-linker, STEM tomography revealed a honeycomb superstructure constructed by hexagonal close-packed cylinders of the smaller POSS cluster and an orderly arranged framework of the larger POM cluster either in single or bicrystals. Within the hexagonal superstructure, the POM clusters form a bilayer arrangement as basic structural motive (Fig. 1). However, locally significant structural variations are observed, both in terms of the POM neighbor coordination (Fig. $1 \mathrm{E}$ ) as well as for the tessellation of the supercell arrangement (Fig. 1F). This self-organization is the combined effect of phase separation of the two immiscible blocks favoring a hexagonal framework, while ordered packing of the POM clusters favors an extended bilayer leading to a geometrically frustrated structure. Close to the growth surface, this frustration leads to large structural variations with an increasing number of clusters in the basic motive, while it becomes more defined close to the core of the nanoparticles (Fig. 1D).

With a stiff azo-linker, STEM analysis reveals are more complex structural arrangement. Low-dose cryo STEM tomography and of individual superlattice crystals confirms the self-assembly in a cubic structure with lattice parameters of $\sim 14 \mathrm{~nm}$ in case of the trans configuration (Fig. 2) and $\sim 18 \mathrm{~nm}$ in case of the cis isomer. The high quality 3D tomographic reconstruction enables a mesoscale crystallographic analysis confirming an ordered bicontinuous double diamond (OBDD) structure as only allowed reflections for an OBDD structure with reflection $u p h^{2}+k^{2}+l^{2}=9$ are observed. Very commonly the OBDD lattice forms twined and 5-fold twined superlattice crystals. Furthermore, with the reduced constraints at the surface of the superlattice crystals, the outer two unit cells exhibit a significantly different structure compare to the bulk, explaining why the OBDD structure is only observed for superlattice crystals larger than $\sim 500 \mathrm{~nm}$ diameter.

This presentation will cover both methodology aspects of electron tomography and beam damage effects in low-dose (cryo) STEM as well as the structural aspects of these self-assembled Janus-type molecules [2].

\section{References:}

[1] C. Ma et al, Angew. Chemie. Int. Ed. Engl 54, (2015), p.15699.

[2] The authors acknowledge funding by the National Natural Science Foundation of China for grants (21174080, 21274069, 21334003, and 21422403), the Open Research Fund of State Key Laboratory of Polymer Physics and Chemistry, Changchun Institute of Applied Chemistry and the 
Karlsruhe Nano Micro Facility (KNMF) for access to the electron microscopic equipment.
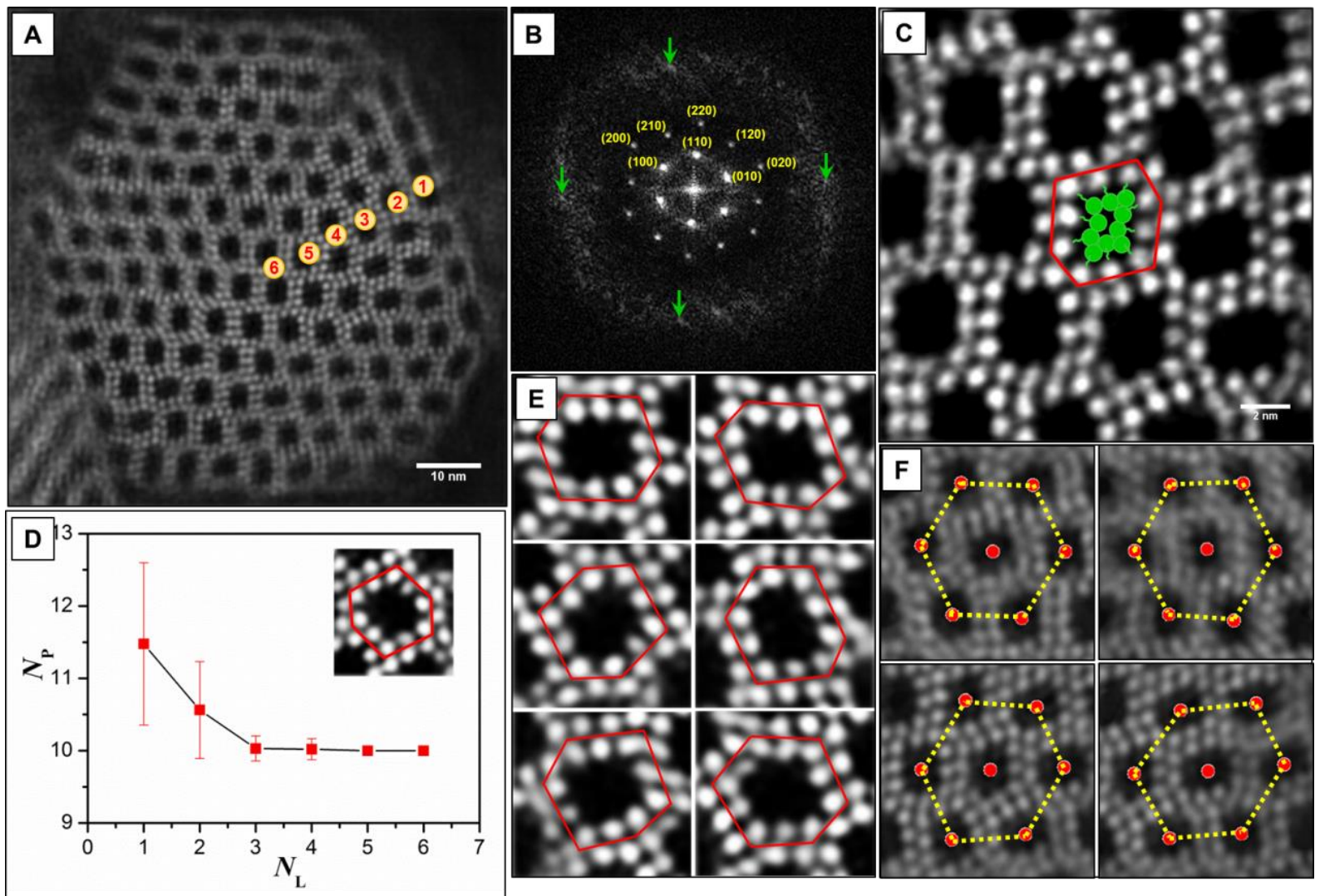

Figure 1. a) Low-dose HRSTEM analysis of self-assembled POM-POSS co-clusters, b) FFT revealing on average a hexagonal ordering, c) detail with molecular sketch superimposed, d) correlation between nearest neighbors and distance from surface, e) local variations of POM assembly, f) local variations of supercell (from [1]).
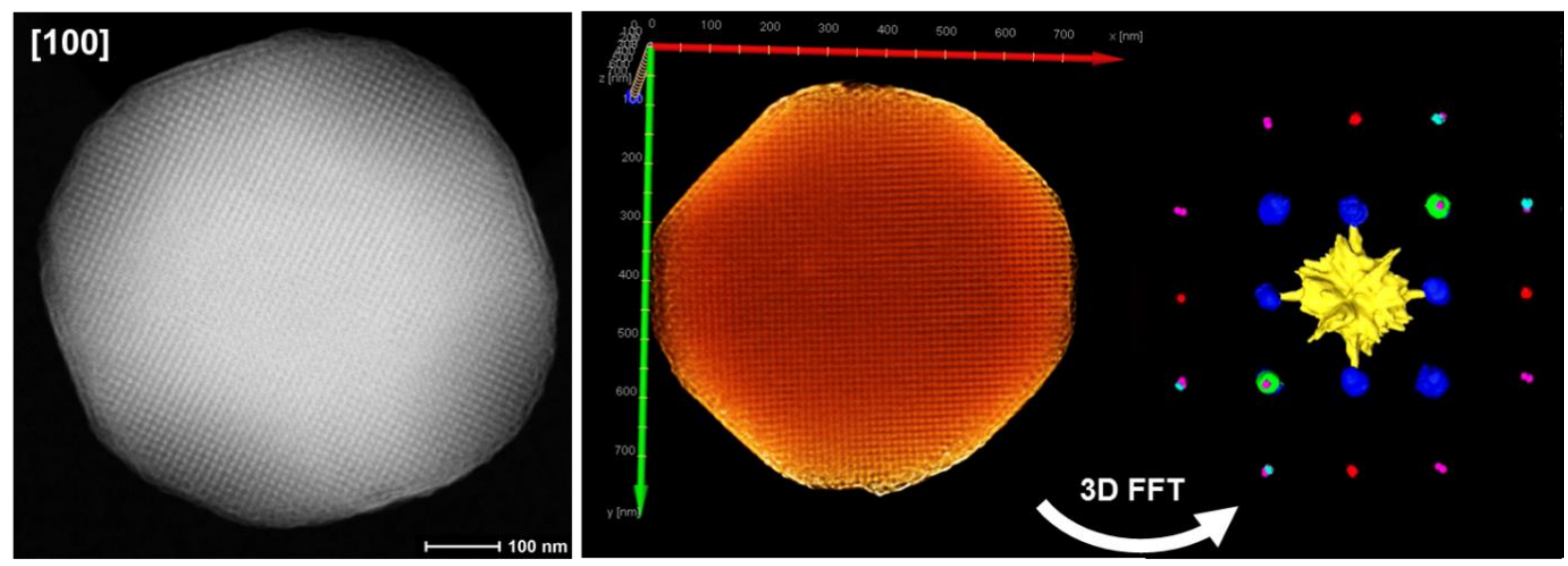

Figure 2. a) Low-dose STEM analysis of self-assembled POM-POSS co-clusters imaged in [100] orientation, b) volume rendering of the corresponding electron tomographic reconstruction, c) 3D FFT of the electron tomographic reconstruction showing the different allowed reflections of an OBDD structure $-\{110\},\{111\},\{200\},\{211\}$ and $\{221\}$. 